\title{
Educação Financeira: Tomar Decisão a Partir da Comparação de Dados de Amortização
}

\section{Paulo Victor Delgado Pereira}

Centro de Ensino Superior de Juiz de Fora - CES/JF

pvparusia@yahoo.com.br

\section{Chang Kuo Rodrigues}

Universidade do Grande Rio - Unigranrio / CES-JF

chang@powerline.com.br

\author{
Denise Mansoldo Salazar \\ Colégio Cristo Redentor \\ dsalazar@powerline.com.br
}

\begin{abstract}
Resumo
Neste artigo, analisaremos os tipos de amortização mais usados na atualidade, os quais são: Sistema de Amortização Francês usando a Tabela Price; o Sistema de Amortização Constante (SAC); e o Sistema de Amortização Misto (SAM). Apresentaremos como se desenvolve o cálculo das amortizações, dos juros e das prestações de cada sistema e as análises de financiamento. Examinaremos ainda as vantagens e as desvantagens de cada um deles, o que influencia na escolha apropriada por um ou outro tipo de transação financeira, quando é necessário contratá-la. Conhecer os procedimentos e as técnicas de amortização definirá, sobretudo, a postura que o indivíduo irá adotar, ao tomar decisões relativas à sua atuação consciente no mundo que o cerca. Esta pesquisa apresenta, portanto, ferramentas para uma discussão mais refinada sobre a Educação Financeira.
\end{abstract}

Palavras-chave: Educação Financeira. Amortização. Movimentação Financeira. Juros.

\section{Financial Education: Comparing the Amortization of the Data to Make Decision}

\begin{abstract}
In this paper, we will examine the types of depreciation over used today, which are: Amortization System using French Table Price; Constant Amortization System (CAS), and the System of Joint Amortization (SJA). We will present how to develop the calculation of depreciation, interest and benefits of each system and funding analyzes. Also examine the advantages and disadvantages of each, which influences the appropriate choice for one or other type of financial transaction when it is necessary to hire. Knowing the procedures and techniques amortization define, especially the posture that the individual will adopt when making decisions regarding their conscious action in the world around him. This research therefore presents tools for a more refined discussion on Financial Education.
\end{abstract}

Keywords: Financial Education. Amortization. Financial Transactions. Interest. 


\section{Introdução}

A Educação Financeira vem firmando suas concepções diante da emergência em se educar pessoas, segundo Jares (2005), para um mundo pós-moderno comandado pela globalização neoliberal e, de certa forma, está presente na vida da grande maioria das pessoas, direta ou indiretamente. Contudo, é fato que nem todos sabem as causas pelas quais uma determinada dívida, ou lucro, incide em uma série de cálculos e conceitos. Seguindo essa motivação, acreditamos que este trabalho poderá servir como fonte para a compreensão dos procedimentos que envolvem uma determinada operação financeira, em uma sociedade ditada pelas regras do sistema econômico ocidental.

Atualmente, o uso das tecnologias permite o acesso imediato a termos como, por exemplo, o fator multiplicativo de uma determinada operação financeira, isentando as pessoas de conhecer os aspectos técnicos que envolvem os cálculos. No entanto, o conhecimento sobre essas técnicas pode, efetivamente, estimular o pensamento crítico, de tal forma que impeça o uso abusivo desse conhecimento.

Assim, nessa direção, esta pesquisa objetiva sugerir que a tecnologia, de fácil acesso a todas as pessoas, não pode ser desprezada quando se pensa em contrair um empréstimo financeiro, em instituições financeiras ou bancárias, de empréstimos ou financiamentos de casa própria, automóveis etc.

O presente trabalho trata, especificamente, da amortização financeira, um conceito que não é usualmente abordado na Educação Básica, sendo mais explorado no Ensino Superior, em cursos diretamente relacionados a esse tema. E, para abordar tal assunto, optou-se por dividir o artigo em quatro seções, a saber: a primeira, introdutória, com o intuito de situar o leitor sobre o tema; a segunda relaciona-se diretamente ao conceito de amortização e de suas particularidades, permitindo, inclusive, uma discussão sobre que tipo de transação financeira é mais adequada a um determinado contexto; na terceira, comparam-se três tipos de sistema financeiro, de modo que se amplie mais ainda a reflexão sobre o tema; e, por fim, a quarta seção está destinada a pontuar algumas considerações conclusivas sobre este trabalho.

Partindo dessa proposta, espera-se que esta investigação proporcione momentos de reflexão que possam intervir com clareza (matemática) nas tomadas de decisão diante de movimentações financeiras.

\section{Amortização Financeira}

Em termos de financiamento, segundo Samanez (2010), a amortização é um processo pelo qual uma dívida ou obrigação é paga gradualmente, por meios de parcelas, de modo que, ao final do prazo estipulado, o valor contratado esteja quitado. Essas parcelas ou prestações são a soma de duas partes, as quais são: a primeira, a parcela de amortização, que é a devolução das partes do valor 
principal, ou seja, o capital emprestado; e a segunda é a parcela de juros correspondentes aos saldos do empréstimo ainda não amortizados. Portanto, é dessa forma que uma prestação é definida:

$$
\text { PRESTAÇÃO = AMORTIZAÇÃO + JUROS }
$$

Essa separação permite discriminar o que representa a devolução do principal (amortização), ou o valor inicial, e o que representam os serviços da dívida (juros). Nos sistemas de amortização, os juros serão calculados sempre sobre o saldo devedor e o não pagamento desses juros em um dado período acarretará em um aumento neste saldo devedor, fazendo com que o mutuário (devedor) venha a pagar juros nas prestações.

Nos sistemas de amortização, podem-se ter, ou não, prazos de carência, que correspondem ao período compreendido entre o prazo de utilização do empréstimo e o pagamento da primeira prestação. Em geral, esse prazo é negociado entre o mutuante (credor) e o mutuário (devedor). No prazo de carência, os juros podem ser capitalizados durante esse prazo ou podem ser pagos, dependendo do acerto entre as partes envolvidas.

Samanez (2010) diz que entre os principais e mais utilizados sistemas de amortização de empréstimos, tem-se: o Sistema de Amortização Francês, conhecido, também, como Sistema Price, que consiste em prestações iguais entre si e calculadas de tal modo que uma parte paga os juros e a outra, o principal; os Sistemas de Amortização Constante (SAC) consistem em parcelas de amortização iguais entre si, em que os juros são calculados a cada período, multiplicando-se a taxa de juros contratada pelo saldo devedor existente, no período anterior; e o Sistema Misto (SAM) é a junção do Sistema Price e o SAC. O SAM é um plano de pagamento composto pela média aritmética calculada das prestações dos planos SAC e Price, sendo que os respectivos prazos e juros seguem uma mesma metodologia.

Além desses sistemas, muitas vezes os bancos e as instituições financeiras criam sistemas de amortização específicos, não-convencionais, adequados a determinadas situações ou características do mercado ou dos clientes. Mas, como este trabalho enfoca apenas os três sistemas apresentados, não discutiremos nenhum outro tipo particular de operacionalização.

Seguem algumas definições de termos de uso corrente, para maior clareza posterior:

a) Mutuante ou credor: aquele que dá o empréstimo.

b) Mutuário ou devedor: aquele que recebe o empréstimo.

c) Taxa de juros: é a taxa de juros contratada entre as partes. Pode referir-se ao custo efetivo do empréstimo ou não, dependendo das condições adotadas.

d) Prazo de utilização: corresponde ao intervalo de tempo durante o qual o empréstimo é transferido do credor para o devedor. Caso o empréstimo seja transferido em uma só parcela, esse prazo é dito unitário.

e) Prazo de amortização: é o intervalo de tempo, durante o qual são pagas as amortizações. 
f) Planilha ou Tabela: é um quadro, padronizado ou não, onde são colocados os valores referentes ao empréstimo, ou seja, cronograma dos valores de recebimento e de pagamento.

g) Prazo total do financiamento: é a soma do prazo de carência com o prazo de amortização.

(MATHIAS, 2002, p.308)

Os termos citados favorecem a compreensão das técnicas específicas da Matemática Financeira e, a partir dessa compreensão, intervir na Educação Financeira, fortalecendo uma prática voltada para o processo de ensino e de aprendizagem em todos os níveis da Educação.

\section{O Sistema Francês de Amortização (Tabela PRICE)}

O Sistema Francês de Amortização é um dos mais usados no Brasil e largamente utilizado nos setores financeiros. Samanez (2010) fez a seguinte afirmação a respeito desse Sistema:

[...] a Tabela Price é um caso particular e muito utilizado do Sistema Francês de Amortização em que a taxa de juros é dada em termos nominais (na prática, em termos anuais) e as prestações têm período menor que aquele a que a taxa de juros se refere (em geral as amortizações são feitas em base mensal). Nesse sistema, o cálculo das prestações é feito usando-se a taxa proporcional ao período a que se refere a prestação, calculada a partir da taxa nominal. (SAMANEZ, 2010, p.156)

Esse sistema, conforme já mencionado, é o mais utilizado pelas instituições financeiras e pelo comércio em geral, e é também conhecido no Brasil como "Sistema da Tabela Price" ou, simplesmente, “Tabela Price”. (SAMANEZ, 2010; MATHIAS, 2002)

A denominação "Tabela Price" veio do matemático e pensador inglês Richard Price (17231791), o qual incorporou a teoria dos juros compostos às amortizações de empréstimos (ou financiamentos). Apesar de o sistema ter sido criado por um inglês, leva a denominação de “Sistema Francês” por se desenvolver efetivamente na França, no Século XIX. Sobrinho (2000) diz que:

\footnotetext{
Esse sistema consiste em um plano de amortização de uma dívida em prestações periódicas, iguais e sucessivas, dentro do conceito de termos vencidos, em que o valor da prestação, ou pagamento, é composto de duas parcelas distintas: uma de juros e outra de capital (chamada amortização). (SOBRINHO, 2000, p.220-221)
}

Ainda com relação ao Sistema Francês (ou Tabela Price), as prestações podem variar temporalmente, isto é, podem ser mensais, trimestrais, semestrais ou anuais, contudo, devem seguir o mesmo padrão, em termos de vencimento.

As prestações são obtidas com base na mesma fórmula utilizada para séries de pagamentos iguais com termos vencidos, ou seja, no vencimento (ou postecipados), usando as taxas diversificadas da Tabela Price, isto é, seguindo as notações de Samanez (2010): 


$$
R=P\left[\frac{(1+i)^{n} \times i}{(1+i)^{n}-1}\right] \quad \text { ou } \quad R=P \times F R C(i, n)
$$

Sendo que $R$ é a parcela a ser paga; $P$, o principal; e, $i$, a taxa de juro em um determinado período, $n$. Vale retomar que a parcela de juros é obtida multiplicando-se a taxa de juros (mensal, trimestral, semestral ou anual) pelo saldo devedor existente no final do período imediatamente anterior (mês, trimestre, semestre ou ano); a parcela de amortização é determinada pela diferença entre o valor da prestação e o valor da parcela de juros. Portanto, o valor da parcela de juros referente à primeira prestação de uma série de pagamentos mensais é igual à taxa multiplicada pelo valor do capital emprestado ou financiado.

Exemplificando essa situação, descrevemos como calcular os valores das parcelas de juros e amortização referentes à primeira prestação, de um empréstimo de $\mathrm{R} \$ 8.530,20$, à taxa de $3 \%$ ao mês, para ser liquidado em 10 prestações iguais.

Vejamos o cálculo de R, de acordo com a seguinte Tabela A, Tabela de FRC:

Tabela A - Tabela de FRC

\begin{tabular}{|c|c|c|c|}
\hline \multicolumn{4}{|c|}{ Série Uniforme de Pagamento(FRC) } \\
\hline $\mathrm{n}$ & $1 \%$ & $2 \%$ & $3 \%$ \\
\hline 1 & 1,010000 & 1,020000 & 1,030000 \\
\hline 2 & 0,507512 & 0,515050 & 0,522611 \\
\hline 3 & 0,340022 & 0,346755 & 0,353530 \\
\hline 4 & 0,256281 & 0,262624 & 0,269027 \\
\hline 5 & 0,206040 & 0,212158 & 0,218355 \\
\hline 6 & 0,172548 & 0,178526 & 0,184598 \\
\hline 7 & 0,148628 & 0,154512 & 0,160506 \\
\hline 8 & 0,130690 & 0,136510 & 0,142456 \\
\hline 9 & 0,116740 & 0,125150 & 0,128434 \\
\hline 10 & 0,105582 & 0,111327 & 0,117231 \\
\hline 11 & 0,096454 & 0,102178 & 0,108077 \\
\hline 12 & 0,888490 & 0,094560 & 0,100462 \\
\hline 13 & 0,824150 & 0,088118 & 0,094030 \\
\hline 14 & 0,769010 & 0,082602 & 0,088526 \\
\hline 15 & 0,072124 & 0,077825 & 0,083767 \\
\hline \multicolumn{3}{|c|}{ Fonte: ARAUUJO,1993, p.295-297 } \\
\hline
\end{tabular}

$$
\begin{gathered}
R=P \times F R C(i, n)=8.530,20 \times \operatorname{FRC}(3 \%, 10) \\
\mathrm{R}=8.530,20 \times 0,11723=1.000,00
\end{gathered}
$$

O valor da parcela de juros $(\mathrm{J})$ será de:

$$
I=i \times P=0,03 \times 8.530,20=255,91
$$

E o valor da parcela de amortização (A):

$$
A=R-J=1.000,00-255,91=744,09
$$


Mas, para que possamos achar as demais parcelas de juros e as de amortização das prestações seguintes, precisamos adotar as seguintes notações:

$\mathrm{J}_{\mathrm{t}}=$ parcela de juros referente ao período de ordem $\mathrm{t}(\mathrm{t}=1,2,3, \ldots, \mathrm{n})$.

$\mathrm{A}_{\mathrm{t}}=$ parcela de amortização referente à prestação de ordem $\mathrm{t}(\mathrm{t}=1,2,3, \ldots, \mathrm{n})$.

$\mathrm{P}_{\mathrm{t}}=$ Saldo devedor referente ao período de ordem $\mathrm{t}(\mathrm{t}=0,1,2,3, \ldots, \mathrm{n}-1)$.

Portanto, a segunda prestação será calculada da seguinte forma:

$$
\begin{gathered}
I_{2}=i \times P_{\mathbf{1}} \\
\mathbf{P}_{\mathbf{1}}=\mathbf{P}_{\mathbf{0}}-\mathbf{A}_{\mathbf{1}}=\mathbf{8 . 5 3 0 , 2 0 - 7 4 4 , 0 9 = 7 . 7 8 6 , 1 1} \\
I_{\mathbf{2}}=0,03 \times 7.786,11=233,5 \mathbf{8} \\
A_{\mathbf{2}}=R-J_{\mathbf{2}}=1.000,00-233,58=766,42
\end{gathered}
$$

Para a terceira prestação, ou terceira amortização:

$$
\begin{aligned}
& I_{3}=i \times P_{\mathbf{2}} \\
& \mathbf{P}_{2}=\mathbf{P}_{\mathbf{1}}-\mathbf{A}_{2}=7.786,11-766,42=7.019,69 \\
& I_{\mathbf{3}}=0,03 \times 7.7019,69=210,59
\end{aligned}
$$

\begin{tabular}{|c|c|c|c|c|c|c|c|c|}
\hline $\mathrm{t}$ & \multicolumn{2}{|c|}{$\begin{array}{c}\text { Saldo Devedor } \\
\left(\mathrm{P}_{\mathrm{t}}\right)\end{array}$} & \multicolumn{2}{|c|}{$\begin{array}{c}\text { Amortização } \\
\left(A_{t}\right)\end{array}$} & \multicolumn{2}{|c|}{$\begin{array}{c}\text { Juros } \\
\left(\mathrm{J}_{\mathrm{t}}\right)\end{array}$} & \multicolumn{2}{|c|}{$\begin{array}{c}\text { Prestação } \\
\text { ( R) }\end{array}$} \\
\hline 0 & $\mathrm{R} \$$ & $8.530,20$ & & - & & - & & - \\
\hline 1 & $\mathrm{R} \$$ & $7.786,11$ & $\mathrm{R} \$$ & 744,09 & $\mathrm{R} \$$ & 255,91 & $\mathrm{R} \$$ & $1.000,00$ \\
\hline 2 & $\mathrm{R} \$$ & $7.019,69$ & $\mathrm{R}$ & 766,42 & $\mathrm{R} \$$ & 233,58 & $\mathrm{R} \$$ & $1.000,00$ \\
\hline 3 & $\mathrm{R} \$$ & $6.230,28$ & $\mathrm{R} \$$ & 789,41 & R\$ & 210,59 & $\mathrm{R} \$$ & $1.000,00$ \\
\hline 4 & $\mathrm{R} \$$ & $5.417,19$ & $\mathrm{R} \$$ & 813,09 & R\$ & 186,91 & $\mathrm{R} \$$ & $1.000,00$ \\
\hline 5 & $\mathrm{R} \$$ & $4.579,71$ & $\mathrm{R} \$$ & 837,48 & R\$ & 162,52 & $\mathrm{R} \$$ & $1.000,00$ \\
\hline 6 & $\mathrm{R} \$$ & $3.717,10$ & $\mathrm{R} \$$ & 862,61 & R\$ & 137,39 & $\mathrm{R} \$$ & $1.000,00$ \\
\hline 7 & $\mathrm{R} \$$ & $2.828,61$ & $\mathrm{R} \$$ & 888,49 & $\mathrm{R} \$$ & 111,51 & $\mathrm{R} \$$ & $1.000,00$ \\
\hline 8 & $\mathrm{R} \$$ & $1.913,47$ & $\mathrm{R} \$$ & 915,14 & $\mathrm{R} \$$ & 84,86 & $\mathrm{R} \$$ & $1.000,00$ \\
\hline 9 & $\mathrm{R} \$$ & 970,87 & $\mathrm{R} \$$ & 942,60 & $\mathrm{R} \$$ & 57,40 & $\mathrm{R} \$$ & $1.000,00$ \\
\hline 10 & & - & $\mathrm{R} \$$ & 970,87 & $\mathrm{R} \$$ & 29,13 & $\mathrm{R} \$$ & $1.000,00$ \\
\hline TOTAL & & - & $\mathrm{R} \$$ & $8.530,20$ & $\mathrm{R} \$$ & $1.469,80$ & $\mathrm{R} \$$ & $10.000,00$ \\
\hline
\end{tabular}

Se operarmos da mesma forma para outras prestações, teremos os valores abaixo (para série de 10 prestações), como podemos ver na Tabela 1:

Tabela 1- Valores para série de 10 prestações (Tabela Price).

Como podemos perceber, na Tabela 1, o valor da prestação (R) é a mesma em todas as parcelas, mas podemos notar que o valor da parcela de amortização $\left(A_{t}\right)$ vai aumentando no decorrer do tempo, enquanto o valor da parcela de juros $\left(\mathrm{J}_{\mathrm{t}}\right)$ vai diminuindo.

Nesse contexto, a representação gráfica deste sistema, em que as prestações são fixas com as amortizações crescendo e os juros começando alto e caindo no decorrer do período, pode ser representada segundo a Figura 1. 


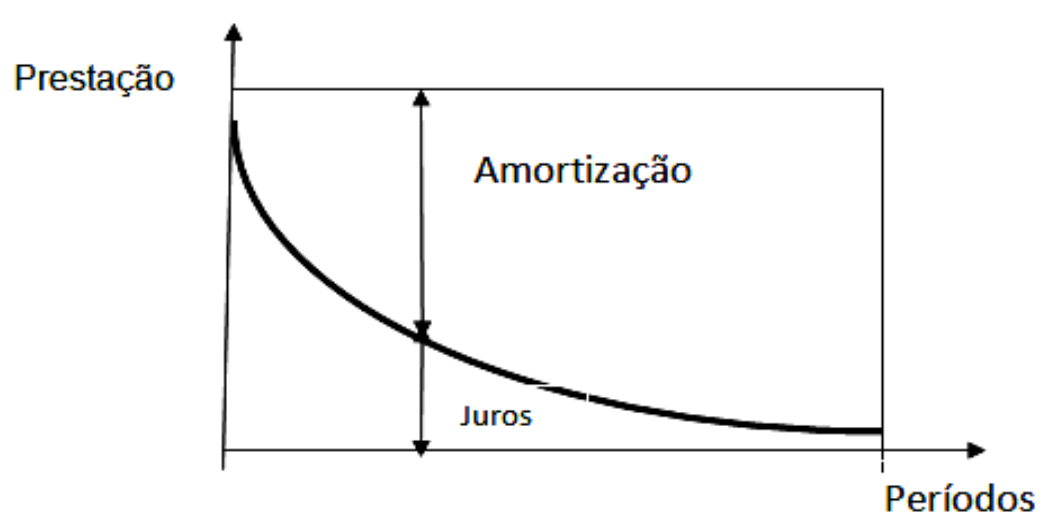

Fonte: MATHIAS, 2002, p.309

Como já foi analisado e visto anteriormente na Tabela 1, podemos observar, no gráfico, que as prestações no eixo das abscissas seguem constantes no decorrer do período; enquanto a parcela de amortização cresce, os juros abaixam, a cada período.

\section{Sistema de Amortização Constante (SAC)}

O Sistema de Amortização Constante (SAC) é um sistema que sempre terá a amortização constante fazendo com que os juros abaixem no decorrer de cada período, diferentemente da Tabela Price, que tem suas prestações constantes, sendo que as amortizações crescem à medida que o prazo aumenta. Sobrinho (2000) diz que:

O SAC consiste em um plano de amortização de uma divida em prestações periódicas, sucessiva e decrescente em progressão aritmética, dentro do conceito de termos vencidos, em que o valor de cada prestação é composto por uma parcela de juros e outra de parcela capital (ou amortização). (SOBRINHO, 2000, p.230)

A parcela de amortização é calculada dividindo o valor do empréstimo (ou financiamento) pelo número de prestações, enquanto a de juros se consegue multiplicando a taxa de juros pelo saldo devedor existente anteriormente.

Vamos verificar um exemplo de como se procede a esse sistema ao elaborar um plano de pagamento, com base no Sistema de Amortização constante, correspondente a um empréstimo de R \$ $100.000,00$, à taxa de $3 \%$ ao mês, a ser liquidado em 10 prestações mensais. A amortização é constante, pois foi obtida pelo quociente entre o principal e o período estipulado.

$$
\mathrm{A}=\frac{P_{0}}{n}=\frac{100.000,00}{10}=10.000,00
$$

A $1^{\text {a }}$ prestação é adquirida da seguinte forma:

$$
R_{1}=A+J_{1}=10.000,00+0,03 \times 100.000,00 \quad R_{1}=10.000,00+3.000,00=
$$


A $2^{\mathrm{a}}$ prestação segue.

$$
\begin{gathered}
R_{\mathbf{z}}=A+J_{\mathbf{z}}=10.000,00+0,03 \times 90.000,00 . \\
R_{2}=10.000,00+2.700,00=12.700,00
\end{gathered}
$$

Aqui o valor de R\$ 90.000,00 refere-se ao saldo devedor existente no período imediatamente anterior, após o pagamento da $1^{\mathrm{a}}$ parcela de amortização, de R $\$ 10.000,00$.

A $3^{\circ}$ parcela é calculada do mesmo modo.

$$
\begin{gathered}
R_{\mathrm{a}}=A+J_{\mathrm{a}}=10.000,00+0,03 \times 80.000,00 . \\
R_{3}=10.000,00+2.400,00=12.400,00
\end{gathered}
$$

E assim ocorre, sucessivamente, até a $10^{\mathrm{a}}$ prestação.

\begin{tabular}{|c|c|c|c|c|}
\hline & Saldo Devedor & Amortizações Constantes & Juros & Prestação \\
\hline $\mathrm{t}$ & $\left(P_{t}\right)$ & $\left(A_{t}\right)$ & $\left(J_{t}\right)$ & $\left(R_{t}\right)$ \\
\hline 0 & $100.000,00$ & - & - & - \\
\hline$\frac{1}{1}$ & $90.000,00$ & $10.000,00$ & $3.000,00$ & $13.000,00$ \\
\hline 2 & $80.000,00$ & $10.000,00$ & $2.700,00$ & $12.700,00$ \\
\hline 3 & $70.000,00$ & $10.000,00$ & $2.400,00$ & $12.400,00$ \\
\hline 4 & $60.000,00$ & $10.000,00$ & $2.100,00$ & $12.100,00$ \\
\hline 5 & $50.000,00$ & $10.000,00$ & $1.800,00$ & $11.800,00$ \\
\hline 6 & $40.000,00$ & $10.000,00$ & $1.500,00$ & $11.500,00$ \\
\hline 7 & $30.000,00$ & $10.000,00$ & $1.200,00$ & $11.200,00$ \\
\hline 8 & $20.000,00$ & $10.000,00$ & 900,00 & $10.900,00$ \\
\hline 9 & $10.000,00$ & $10.000,00$ & 600,00 & $10.600,00$ \\
\hline 10 & - & $10.000,00$ & 300,00 & $10.300,00$ \\
\hline TOTAL & - & $100.000,00$ & $16.500,00$ & $116.500,00$ \\
\hline
\end{tabular}

A Tabela 2, a seguir, apresenta o plano de pagamento das prestações desdobradas, especificando amortização e juros.

Tabela 2 - Valores para série de 10 prestações (SAC)

Podemos observar, na tabela, que as prestações decrescem em uma razão constante de R\$ 300,00, devido à multiplicação da taxa pela amortização constante, ou seja, $0,03 \times 10.000,00=300,00$.

Vejamos a representação gráfica desse caso, Figura 2, em que a amortização é constante e os juros vão diminuindo, no decorrer do período. 


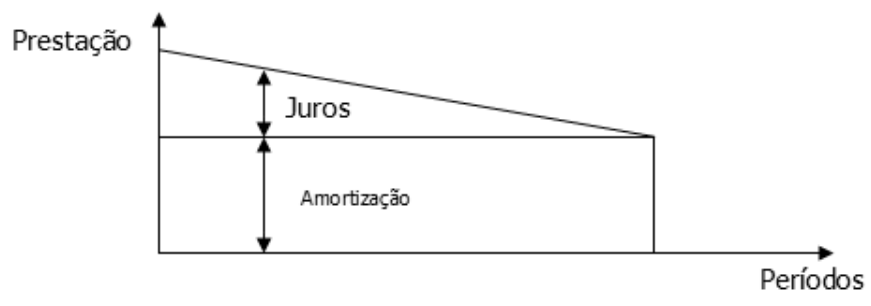

Fonte: MATHIAS, 2002, p.309

A partir desse gráfico, a prestação, nesse sistema, vai diminuindo no decorrer do período, enquanto a amortização segue constante e os juros decrescem, pois são calculados a partir do saldo devedor do período anterior.

Vejamos, agora, algumas possibilidades associadas a tal modo de se fazer amortização de dívidas.

I - SAC - com prazo de carência e prazo de utilização unitário

Utilizaremos um exemplo dado por Mathias (2002), para esclarecer a prática do Sistema de Amortização Constante com prazo de carência e prazo de utilização unitário, conforme segue o exemplo a seguir: uma empresa pede emprestado $\mathrm{R} \$ 100.000,00$, e o banco entrega a quantia no ato, concedendo 3 anos de carência (sendo os juros pagos anualmente), taxa de juros de $10 \%$ ao ano e o principal será amortizado em 4 parcelas anuais.

Observemos a planilha a seguir, para verificarmos que a amortização anual será de:

$$
\frac{100.000,00}{4}=25.000,00
$$

Vamos imaginar que o principal fosse emprestado no início do primeiro ano e que as prestações e os juros fossem pagos ao fim de cada ano, conforme os dados da Tabela 3.

\begin{tabular}{|c|c|c|c|c|c|}
\hline $\begin{array}{l}\text { Ano } \\
(\mathrm{t})\end{array}$ & Saque & $\begin{array}{l}\text { Saldo } \\
\text { Devedor } \\
\left(S d_{t}\right)\end{array}$ & $\begin{array}{l}\text { Amortização } \\
\left(A_{t}\right)\end{array}$ & $\begin{array}{l}\text { Juros } \\
\left(U_{t}\right)\end{array}$ & $\begin{array}{l}\text { Prestação } \\
\left(A_{t}+J_{t}\right)\end{array}$ \\
\hline 0 & $100.000,00$ & $100.000,00$ & - & - & - \\
\hline 1 & - & $100.000,00$ & - & $10.000,00$ & $10.000,00$ \\
\hline$\frac{\infty}{2}$ & - & $100.000,00$ & - & $10.000,00$ & $10.000,00$ \\
\hline 3 & - & $75.000,00$ & $25.000,00$ & $10.000,00$ & $35.000,00$ \\
\hline 4 & - & $50.000,00$ & $25.000,00$ & $7.500,00$ & $32.500,00$ \\
\hline 5 & - & $25.000,00$ & $25.000,00$ & $5.000,00$ & $30.000,00$ \\
\hline 6 & - & - & $25.000,00$ & $2.500,00$ & $27.500,00$ \\
\hline Total & - & - & $100.000,00$ & $45.000,00$ & $145.000,00$ \\
\hline
\end{tabular}

Vale observar que, do começo do primeiro ano (data zero), ao fim do terceiro ano, temos 3 períodos, que correspondem à carência. Terminado o período de carência, teremos a primeira parcela de amortização e os juros serão calculados sobre o saldo devedor anterior.

Ou seja, sendo, $J_{t}$ o juro devido no período $\mathrm{t}$, sendo $\mathrm{i}$ a taxa de juros, e $\mathrm{Sd}_{\mathrm{t}-1}$ o saldo devedor do período anterior, temos: 


$$
\mathrm{J}_{\mathrm{t}}=\mathrm{iSd} \mathrm{d}_{\mathrm{t}-1}
$$

Assim, pudemos constatar, no exemplo, que o juro do período foi calculado multiplicandose a taxa (na forma unitária) pelo saldo devedor do período anterior, e a prestação foi obtida somando a parcela de amortização aos juros.

II - SAC - com prazo de carência, juros capitalizados e prazo de utilização unitário

No caso do Sistema de Amortização Constante (SAC) com os juros capitalizados e prazo de utilização unitário, em alguns casos, a empresa pode combinar, ou não, o pagamento dos juros durante o período de carência. Diz-se, então, que os juros foram capitalizados durante a carência.

Podemos ter dois casos, a saber: no primeiro, as amortizações são calculadas em relação ao valor inicial emprestado e os juros capitalizados são pagos no primeiro ano de amortização; no segundo caso, as amortizações são calculadas em relação ao valor inicial emprestado, mais os juros capitalizados durante a carência.

Tomando como base o exemplo anterior, tem-se, conforme descrição da Tabela 4:

Tabela 4 - Situação Caso A

\begin{tabular}{|c|c|c|c|c|c|}
\hline $\begin{array}{c}\text { Ano } \\
(\mathrm{t})\end{array}$ & Saque & $\begin{array}{c}\text { Saldo } \\
\text { Devedor } \\
\left(S d_{t}\right)\end{array}$ & $\begin{array}{l}\text { Amortização } \\
\left(A_{t}\right)\end{array}$ & $\begin{array}{l}\text { Juros } \\
\left.J_{t}\right)\end{array}$ & $\begin{array}{l}\text { Prestação } \\
\left(A_{t}+J_{t}\right)\end{array}$ \\
\hline 0 & $100.000,00$ & $100.000,00$ & - & - & - \\
\hline 1 & - & $110.000,00$ & - & - & - \\
\hline 2 & - & $121.000,00$ & - & - & - \\
\hline 3 & - & $75.000,00$ & $25.000,00$ & $33.100,00$ & $58.100,00$ \\
\hline 4 & - & $50.000,00$ & $25.000,00$ & $7.500,00$ & $32.500,00$ \\
\hline 5 & - & $25.000,00$ & $25.000,00$ & $5.000,00$ & $30.000,00$ \\
\hline 6 & - & - & $25.000,00$ & $2.500,00$ & $27.500,00$ \\
\hline 5 & - & - & $100.000,00$ & $48.100,00$ & $148.100,00$ \\
\hline Total & - & Fonte: MATHIAS, $2002, \mathrm{p} .312$
\end{tabular}

Vale destacar, na Tabela 4, que o saldo devedor foi acrescido dos juros devido à taxa calculada de $10 \%$ ao ano. Ao final do $3^{\circ}$ ano, os juros capitalizados foram pagos juntamente com a primeira prestação, o que a deixou mais alta que as demais. 
Tabela 5 - Situação Caso B

\begin{tabular}{|c|c|c|c|c|c|}
\hline $\begin{array}{c}\text { Ano } \\
(\mathrm{t})\end{array}$ & Saque & $\begin{array}{c}\text { Saldo } \\
\text { Devedor } \\
\left(S d_{t}\right)\end{array}$ & $\begin{array}{l}\text { Amortização } \\
\left(A_{t}\right)\end{array}$ & $\begin{array}{l}\text { Juros } \\
\left(J_{t}\right)\end{array}$ & $\begin{array}{c}\text { Prestação } \\
\left(A_{t}+J_{t}\right)\end{array}$ \\
\hline 0 & $100.000,00$ & $100.000,00$ & - & - & - \\
\hline 1 & - & $110.000,00$ & - & - & - \\
\hline 2 & - & $121.000,00$ & - & - & - \\
\hline 2 & - & $99.825,00$ & $33.275,00$ & - & $33.275,00$ \\
\hline 3 & - & $66.550,00$ & $33.275,00$ & $9.982,50$ & $43.257,50$ \\
\hline 4 & - & $33.275,00$ & $33.275,00$ & $6.655,00$ & $39.930,00$ \\
\hline 5 & - & - & $33.275,00$ & $3.327,50$ & $36.602,50$ \\
\hline 6 & - & - & $133.100,00$ & $19.965,00$ & $153.065,00$ \\
\hline 6
\end{tabular}

Fonte: MATHIAS, 2002, p.313

Na Situação Caso B, foram calculados os juros de $10 \%$ sobre o saldo devedor e acrescidos sobre ele mesmo. No $3^{\circ}$ período, o saldo devedor era:

$$
\mathrm{Sd}_{3}=133.100,00
$$

Portanto, as parcelas de amortização serão as seguintes:

$$
A=\frac{133.100,00}{4}=33.275,00
$$

Já o cálculo das demais prestações é igual ao dos casos anteriores.

Fazendo uma comparação no total das prestações de cada caso apresentado, temos valores diferentes para um empréstimo de R \$100.000,00:

$$
\begin{array}{ll}
\text { Exemplo: } & 145.000,00 \\
\text { CASO A: } & 148.100,00 \\
\text { CASO B: } & 153.065,00
\end{array}
$$

Analisando os três casos, podemos observar que o mutuário terá que pagar mais de $50 \%$ do valor de empréstimo, caso resolva fazer uma capitalização igual à do CASO B; seria melhor usar, então, um prazo de carência em que se pague os juros sem a amortização, igual ao exemplo apresentado.

III - SAC - com prazo de carência e prazo de utilização não-unitário

Utilizando, ainda, o exemplo dado por Mathias (2002) no item (I), para o caso do Sistema de Amortização Constante com prazo de carência e prazo de utilização não unitário, mas admitindo que o empréstimo de $\mathrm{R} \$ 100.000,00$ seja dado pelo banco em duas parcelas iguais, defasada em 1 ano, e que as demais condições sejam as mesmas, vejamos os dados da Tabela 6 . 
Tabela 6 - SAC, com carência e prazo não unitánio

\begin{tabular}{|c|c|c|c|c|c|}
\hline $\begin{array}{c}\text { Ano } \\
(\mathrm{t})\end{array}$ & Saque & $\begin{array}{c}\text { Saldo } \\
\text { Devedor } \\
\left(S d_{t}\right)\end{array}$ & $\begin{array}{l}\text { Amortização } \\
\left(A_{t}\right)\end{array}$ & $\begin{array}{l}\text { Juros } \\
\left(J_{t}\right)\end{array}$ & $\begin{array}{l}\text { Prestação } \\
\left(A_{t}+J_{t}\right)\end{array}$ \\
\hline 0 & $50.000,00$ & $50.000,00$ & - & - & - \\
\hline 1 & $50.000,00$ & $100.000,00$ & - & $5.000,00$ & $5.000,00$ \\
\hline 2 & - & $100.000,00$ & - & $10.000,00$ & $10.000,00$ \\
\hline 3 & - & $75.000,00$ & $25.000,00$ & $10.000,00$ & $35.000,00$ \\
\hline 4 & - & $50.000,00$ & $25.000,00$ & $7.500,00$ & $32.500,00$ \\
\hline 4 & - & $25.000,00$ & $25.000,00$ & $5.000,00$ & $30.000,00$ \\
\hline 5 & - & - & $25.000,00$ & $2.500,00$ & $27.500,00$ \\
\hline 6 & - & - & $100.000,00$ & $40.000,00$ & $140.000,00$ \\
\hline Total & - & \multicolumn{7}{|c|}{ Fonte: MATHIAS, 2002, p.314 } \\
\hline \multicolumn{7}{|c|}{} \\
\hline
\end{tabular}

Comparando a Tabela 6 à Tabela 3 do item (I), constatamos que, apesar de ter-se pago juros após o primeiro período, os demais cálculos são os mesmos. Nessas condições, o único efeito de um prazo de utilização não-unitário é gerar um fluxo de prestações mais uniforme e um pagamento de juros, no final, menor.

Apesar de o valor das prestações ser inferior nos exemplos anteriores, não podemos dizer que a taxa também é inferior, pois ela é a mesma, conforme pudemos verificar (de 10\% ao ano).

O valor atual, na data zero, dos desembolsos feitos pelo banco, à taxa de $10 \%$ ao ano, é:

$$
V_{B}=\frac{50.000,00}{(1,10)^{0}}+\frac{50.000,00}{(1,10)^{\mathbf{1}}} \cong 95.454,55
$$

E o valor atual das prestações pagas pelo cliente, nas mesmas condições:

$V_{C}=\frac{5.000,00}{(1,10)^{\mathbf{1}}}+\frac{10.000,00}{(1,10)^{\mathbf{2}}}+\frac{35.000,00}{(1,10)^{\mathbf{3}}}+\frac{32.500,00}{(1,10)^{\mathbf{4}}}+\frac{30.000,00}{(1,10)^{\mathbf{5}}}+\frac{27.500,00}{(1,10)^{\mathbf{6}}} \cong 95.454,55$

Como $V_{B}=V_{C}$, podemos constatar que o cliente, ou devedor, devolveu realmente o que lhe foi emprestado pelo credor, com a devida taxa de juros contratada.

\section{Sistema De Amortização Misto (SAM)}

O Sistema de Amortização Misto também recebe o nome de Sacre (Sistema de amortização crescente) e foi criado pelo BNH (Banco Nacional de Habitação), em maio de 1979, sendo adotado recentemente pelo SFH (Sistema Financeiro de Habitação) para liquidação e financiamento da casa própria. Sobrinho (2000) diz que:

[...] constituiu-se num misto entre o Sistema Francês de Amortização (Tabela Price) e o Sistema de Amortização Constante (SAC), originando-se daí a sua denominação. O SAM é um plano de pagamento composto pela média aritmética calculada das prestações dos planos SAC e Price, sendo que os respectivos prazos e juros seguem nas mesmas condições. (SOBRINHO, 2000, p.239)

No SAM, as amortizações são maiores que as da Tabela Price, aproximadamente até a metade do período de financiamento; após esse tempo, o saldo devedor tem uma queda substancial, e não se corre muito risco de ter resíduo no final do contrato, por isso, é uma das principais 
desvantagens do SAM ter prestações iniciais mais altas que as do Sistema Price. Contudo, após a metade do período, haverá uma grande queda nos valores a serem pagos.

Para obter os valores de um plano, temos que, primeiramente, saber os valores correspondentes nos sistemas SAC e Price e, a seguir, calcular suas respectivas médias aritméticas. Observando o exemplo dado por Sobrinho (2000), podemos ter uma base de como funciona esse sistema, partindo do exemplo dado a seguir: elaborar um plano de pagamento com base no Sistema de Amortização Misto, correspondente a um empréstimo de R\$12.000,00, a uma taxa de 2\% ao mês, a ser liquidado em doze prestações mensais.

\begin{tabular}{|c|c|c|c|c|}
\hline \multirow[b]{2}{*}{$\mathrm{t}$} & \multicolumn{4}{|c|}{ SISTEMA FRANCÊS } \\
\hline & $\mathbf{P}_{t}$ & $\mathbf{A}_{t}$ & $\mathrm{~J}_{\mathrm{t}}$ & $\mathbf{R}_{-}$ \\
\hline 0 & $12.000,00$ & - & - & - \\
\hline$\frac{1}{2}$ & $11.105,28$ & 894,72 & 240,00 & $1.134,72$ \\
\hline 2 & $10.192,67$ & 912,61 & 222,11 & $1.134,72$ \\
\hline 3 & $9.261,80$ & 930,87 & 203,85 & $1.134,72$ \\
\hline 4 & $8.312,32$ & 949,48 & 185,24 & $1.134,72$ \\
\hline 5 & $7.343,85$ & 968,47 & 166,25 & $1.134,72$ \\
\hline 6 & $6.356,01$ & 987,84 & 146,88 & $1.134,72$ \\
\hline 7 & $5.348,41$ & $1.007,60$ & 127,12 & $1.134,72$ \\
\hline 8 & $4.320,66$ & $1.027,75$ & 106,97 & $1.134,72$ \\
\hline 9 & $3.272,35$ & $1.048,31$ & 86,41 & $1.134,72$ \\
\hline 10 & $2.203,08$ & $1.069,27$ & 65,45 & $1.134,72$ \\
\hline 11 & $1.112,42$ & $1.090,66$ & 44,06 & $1.134,72$ \\
\hline 12 & - & $1.112,42$ & 22,30 & $1.134,72$ \\
\hline \multicolumn{2}{|c|}{ TOTAL } & $12.000,00$ & $1.616,64$ & $13.616,64$ \\
\hline
\end{tabular}

\begin{tabular}{|c|c|c|c|c|}
\hline \multirow[b]{2}{*}{$\mathrm{t}$} & \multicolumn{4}{|c|}{ SA } \\
\hline & $\mathbf{P}_{t}$ & $\mathbf{A}_{-}$ & $\mathrm{J}_{\mathrm{t}}$ & $\mathbf{R}_{t}$ \\
\hline 0 & $12.000,00$ & - & - & - \\
\hline 1 & $11.000,00$ & $1.000,00$ & 240,00 & $1.240,00$ \\
\hline 2 & $10.000,00$ & $1.000,00$ & 220,00 & $1.220,00$ \\
\hline 3 & $9.000,00$ & $1.000,00$ & 200,00 & $1.200,00$ \\
\hline 4 & $8.000,00$ & $1.000,00$ & 180,00 & $1.180,00$ \\
\hline 5 & $7.000,00$ & $1.000,00$ & 160,00 & $1.160,00$ \\
\hline 6 & $6.000,00$ & $1.000,00$ & 140,00 & $1.140,00$ \\
\hline 7 & $5.000,00$ & $1.000,00$ & 120,00 & $1.120,00$ \\
\hline 8 & $4.000,00$ & $1.000,00$ & 100,00 & $1.100,00$ \\
\hline 9 & $3.000,00$ & $1.000,00$ & 80,00 & $1.080,00$ \\
\hline 10 & $2.000,00$ & $1.000,00$ & 60,00 & $1.060,00$ \\
\hline 11 & $1.000,00$ & $1.000,00$ & 40,00 & $1.040,00$ \\
\hline 12 & - & $1.000,00$ & 20,00 & $1.020,00$ \\
\hline \multicolumn{2}{|c|}{ TOTAL } & $12.000,00$ & $1.560,00$ & $13.560,00$ \\
\hline
\end{tabular}




\begin{tabular}{|c|c|c|c|c|}
\hline \multirow[b]{2}{*}{$\mathrm{t}$} & \multicolumn{4}{|c|}{ SAM } \\
\hline & $\mathbf{P}_{t}$ & $\mathbf{A}_{t}$ & $\mathrm{Jt}_{\mathrm{t}}$ & $\mathbf{R}_{\mathrm{t}}$ \\
\hline 0 & $12.000,00$ & - & - & - \\
\hline 1 & $11.052,64$ & 947,36 & 240,00 & $1.187,36$ \\
\hline 2 & $10.096,34$ & 956,30 & 221,06 & $1.177,36$ \\
\hline 3 & $9.130,90$ & 965,44 & 201,92 & $1.167,36$ \\
\hline 4 & $8.156,16$ & 974,74 & 182,62 & $1.157,36$ \\
\hline 5 & $7.171,93$ & 984,23 & 163,13 & $1.147,36$ \\
\hline 6 & $6.178,01$ & 993,92 & 143,44 & $1.137,36$ \\
\hline 7 & $5.174,21$ & $1.003,80$ & 123,56 & $1.127,36$ \\
\hline 8 & $4.160,34$ & $1.013,87$ & 103,49 & $1.117,36$ \\
\hline 9 & $3.136,18$ & $1.024,16$ & 83,20 & $1.107,36$ \\
\hline 10 & $2.101,54$ & $1.034,64$ & 62,72 & $1.097,36$ \\
\hline 11 & $1.056,21$ & $1.045,33$ & 42,03 & $1.087,36$ \\
\hline 12 & - & $1.056,21$ & 21,15 & $1.077,36$ \\
\hline \multicolumn{2}{|c|}{ TOTAL } & $12.000,00$ & $1.588,32$ & $13.588,32$ \\
\hline
\end{tabular}

Como podemos verificar nas Tabelas 7, 8 e 9, os valores das prestações dos saldos devedores, das amortizações e dos juros do SAM são realmente a média aritmética dos valores da Tabela Price e do SAC. Observamos, também, que as prestações do SAM decrescem a uma razão constante de R \$10,00, sendo essa a metade da razão do decréscimo do SAC.

Para determinar qualquer valor de um plano de pagamento, sem ter que desenvolver tabelas, é necessário que se conheçam os números referentes ao valor de prestação de ordem $t$.

$$
R_{t}^{(M)}=\frac{R^{(P)}+R_{t}^{(C)}}{2}=\frac{1}{2}\left[R^{(P)}+R_{t}^{(C)}\right] \text {, em que: }
$$

Sendo que:

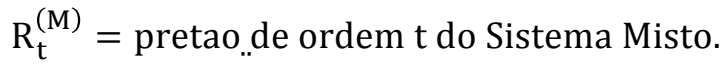

$$
\begin{aligned}
& \mathrm{R}^{(\mathrm{P})}=\text { prestao do Sistema Price (constante). } \\
& \mathrm{R}_{\mathrm{t}}^{(\mathrm{C})}=\text { prestao de ordem } \mathrm{t} \text { do Sistema de Amortizao... Constante. }
\end{aligned}
$$

Portanto, (M) indica tratar-se do Sistema Misto, (P) do Price e (C) do SAC.

$$
R_{t}^{(M)}=\frac{1}{2}\left\{P_{0} \times \operatorname{FRC}(i, n)+A^{(C)}[1+i(n-t+1)]\right\}
$$

Valor da prestação de ordem $\mathrm{t}$ (em função de $\mathrm{R}_{1}$ e r):

$$
\begin{gathered}
R_{t}^{(M)}=R_{1}^{(M)}-(t-1) r^{(M)} \\
r^{(M)}=\frac{r^{(C)}}{2}
\end{gathered}
$$

Valor da parcela de amortização de ordem t:

$$
A_{t}^{(M)}=\frac{1}{2}\left[A_{1}^{(P)}+A^{(C)}\right]
$$




$$
A_{t}^{(M)}=\frac{1}{2}\left[A_{1}^{(P)}(1+i)^{t-1}+\frac{P_{0}}{n}\right]
$$

Saldo devedor em ordem t:

$$
\begin{gathered}
P_{t}^{(M)}=\frac{1}{2}\left[P_{t}^{(P)}+P_{t}^{(C)}\right] \\
P_{t}^{(M)}=\frac{1}{2}\left[R^{(P)} \times F V A(i, n-t)+A^{(C)}(n-t)\right]
\end{gathered}
$$

O saldo devedor de ordem $\mathrm{t}-1$ :

$$
\begin{gathered}
P_{t-1}^{(M)}=\frac{1}{2}\left[P_{t-1}^{(P)}+P_{t-1}^{(C)}\right] \\
P_{t-1}^{(M)}=\frac{1}{2}\left[R^{(P)} \times F V A(i, n-t+1)+A^{(C)}(n-t+1)\right]
\end{gathered}
$$

$\mathrm{O}$ valor da parcela de juros de ordem $\mathrm{t}$ :

$$
\begin{gathered}
I_{t}^{(M)}=i \times P_{t-1}^{(M)}=\frac{1}{2}\left[R^{(P)} \times F V A(i, n-t+1)+A^{(C)}(n-t+1)\right] \\
J_{t}^{(M)}=\frac{1}{2}\left[J_{t}^{(P)}+J_{t}^{(C)}\right]
\end{gathered}
$$

Vejamos exemplos usando algumas das relações apresentadas anteriormente.

Tomando como base o exemplo anterior, dado por Sobrinho $(2000)$, em que $P_{(0)}=$ $R \$ 12.000,00, i=2 \%$ ao $m_{\mathrm{a}} \mathrm{s}$, e $n=12$ e cujas respostas estão na Tabela 9 de representação do SAM, podemos calcular o valor da $9^{\text {a }}$ prestação:

$$
\begin{gathered}
R_{t}^{(M)}=\frac{1}{2}\left\{P_{0} \times \operatorname{FRC}(i, n)+A^{(c)}[1+i(n-t+1)]\right\} \\
R_{9}^{(M)}=\frac{1}{2}\{12.000 \times \operatorname{FRC}(2 \%, 12)+1.000[1+0,02(12-9+1)]\} \\
R_{9}^{(M)}=\frac{1}{2}(1.134,72+1.080)=\frac{2.214,72}{2}=1.107,36
\end{gathered}
$$

Sabendo-se que $R_{1}^{(M)}=R \$ 1.187,36$ e $r^{(C)}=R \$ 20,00$, será calculado o valor da $5^{\text {a }}$ prestação do plano de pagamento, elaborado de acordo com o SAM.

$$
\begin{gathered}
R_{t}^{(M)}=R_{1}^{(M)}-(t-1) r^{(M)} \\
\text { Como } r^{(M)}=\frac{r^{(C)}}{2}, \text { temos: } r^{(M)}=\frac{20}{2}=10 \\
R_{5}^{(M)}=1.187,36-(5-1) \times 10 \\
R_{5}^{(M)}=1.187,36-4 \times 10 \\
R_{5}^{(M)}=1.147,36
\end{gathered}
$$

Agora, determinaremos o valor da parcela de amortização correspondente à $6^{\mathrm{a}}$ prestação. 


$$
\begin{gathered}
A_{t}^{(M)}=\frac{1}{2}\left[A_{1}^{(P)}(1+i)^{t-1}+\frac{P_{0}}{n}\right] \\
A_{1}^{(P)}=R^{(P)}-i \times P_{0} \\
R^{(P)}=P_{0} \times F R C(i, n)=12.000,00 \times F R C(2 \%, 12) \\
R^{(P)}=12.000,00 \times 0,09456=1.134,72 \\
A_{1}^{(P)}=1.134,72-0,02 \times 12.000,00=894,72 \\
A_{6}^{(M)}=\frac{1}{2}\left[894,72(1,02)^{5}+\frac{12.000,00}{12}\right]=\frac{1}{2}(987,84+1.000,00) \\
A_{6}^{(M)}=993.92
\end{gathered}
$$

Calculando, agora, o saldo devedor, após o pagamento da $7^{\text {a }}$ prestação, temos:

$$
\begin{gathered}
P_{t}^{(M)}=\frac{1}{2}\left[R^{(p)} \times F V A(i, n-t)+A^{(c)}(n-t)\right] \\
P_{7}^{(M)}=\frac{1}{2}[1.134,72 \times F V A(2 \%, 5)+1.000,00 \times 5] \\
P_{7}^{(M)}=\frac{1}{2}(1.134,72 \times 4,71346+5.000,00) \\
P_{7}^{(M)}=5.174,21
\end{gathered}
$$

Determinando o valor da parcela de juros referente à $3^{\mathrm{a}}$ prestação:

$$
\begin{gathered}
I_{t}^{(M)}=i \times \frac{1}{2}\left[R^{(P)} \times F V A(i, n-t+1)+A^{(C)}(n-t+1)\right] \\
I_{3}^{(M)}=0,02 \times \frac{1}{2}[1.134,72 \times F V A(2 \%, 10)+1.000,00 \times 10] \\
I_{3}^{(M)}=0,02 \times \frac{1}{2}(1.134,72 \times 8,98259+10.000,00) \\
J_{3}^{(M)}=201,92
\end{gathered}
$$

Como podemos perceber, não é necessária a utilização da Tabela 7, 8 e 9, se utilizarmos as relações apresentadas no exemplo acima.

O Sistema de Amortização Misto (SAM) é mais utilizado nos contratos de financiamentos de imóveis, apesar de muitas construtoras financiarem seus imóveis usando a Tabela Price, mas as instituições bancárias têm preferência pelo SAM.

O SAM foi desenvolvido com o objetivo de permitir maior amortização do valor emprestado, pois em um financiamento de imóvel, a prestação inicial a ser comprometida é de até $30 \%$ da renda comparando com a Tabela Price, em que o comprometimento da renda não pode passar de $25 \%$ da renda; além disso, a prestação no SAM vai diminuindo, conforme observamos nos exemplos anteriores, e, na Tabela Price, a amortização aumenta. 


\section{Análise Comparativa entre os Sistemas Price, SAC e SAM}

Vamos analisar, numericamente, as diferenças entre os três sistemas de amortização trabalhados anteriormente. Com isso, elaboraremos um plano de pagamento para cada um, baseados nos seguintes dados:

- Valor do empréstimo: $\mathrm{R} \$ 200.000,00$;

- Número das prestações mensais: 96;

- Taxa de juros: $1 \%$ ao mês.

Com base na Tabela $\mathrm{B}$ abaixo, podemos verificar que o valor correspondente às prestações do SAC decrescem linearmente de R \$ 4083,33 até R \$ 2104,17, enquanto no Sistema Price as prestações são constantes; já em relação às amortizações, acontece de no SAC elas serem constantes, iguais a R \$2083,33, e no Sistema Price crescerem exponencialmente de R \$1250,57 até $\mathrm{R} \$ 3218,38$. Obviamente, as prestações e as amortizações se igualam em um determinado ponto. Vamos provar isso analiticamente.

\begin{tabular}{|c|c|c|c|c|c|c|c|c|c|c|c|c|}
\hline \multirow[b]{2}{*}{ mes } & \multicolumn{4}{|c|}{ TABELA PRICE } & \multicolumn{4}{|c|}{ SAC } & \multicolumn{4}{|c|}{ SAM } \\
\hline & Saldo devedor & Amortizaçăo & Juros & Prestaçăo & Saldo devedor & Amortizaçăo & Juros & Prestaçăo & Saldo devedor & Amortizaçăo & Juros & Prestaçäo \\
\hline 0 & RS $200.000,00$ & & & & RS $200.000,00$ & & & & RS $200.000,00$ & & & \\
\hline 1 & RS $198.749,43$ & RS $1.250,57$ & RS $2.000,00$ & RS 3.250,57 & RS $197.916,67$ & RS 2.083,33 & RS $2.000,00$ & RS $4.083,33$ & RS $198.333,05$ & RS $1.666,95$ & RS $2.000,00$ & RS 3.666,95 \\
\hline 2 & RS $197.486,36$ & RS $1.263,07$ & RS $1.987,49$ & RS $3.250,57$ & RS $195.833,33$ & RS 2.083,33 & RS $1.979,17$ & RS $4.062,50$ & R\$ $196.659,85$ & RS $1.673,20$ & RS $1.983,33$ & RS 3.656,53 \\
\hline 10 & RS $186.916,29$ & RS $1.367,73$ & RS $1.882,84$ & RS $3.250,57$ & RS $179.166,67$ & RS 2.083,33 & RS $1.812,50$ & RS $3.895,83$ & RS $183.041,48$ & RS $1.725,53$ & RS $1.847,67$ & RS $3.573,20$ \\
\hline 20 & RS $172.463,73$ & RS $1.510,82$ & RS $1.739,75$ & RS $3.250,57$ & RS $158.333,33$ & RS 2.083,33 & RS $1.604,17$ & RS $3.687,50$ & RS $165.398,53$ & RS $1.797,08$ & RS $1.671,96$ & RS $3.469,03$ \\
\hline 30 & RS $156.499,12$ & RS $1.668,89$ & RS $1.581,68$ & RS $3.250,57$ & RS $137.500,00$ & RS 2.083,33 & RS $1.395,83$ & RS $3.479,17$ & RS $146.999,56$ & RS $1.876,11$ & RS $1.488,76$ & RS $3.364,87$ \\
\hline 40 & RS $138.864,25$ & RS $1.843,49$ & RS $1.407,08$ & RS $3.250,57$ & RS $116.666,67$ & RS 2.083,33 & RS $1.187,50$ & RS $3.270,83$ & RS $127.765,46$ & RS $1.963,41$ & RS $1.297,29$ & RS $3.260,70$ \\
\hline 41 & RS $137.002,33$ & RS $1.861,93$ & RS $1.388,64$ & RS $3.250,57$ & RS $114.583,33$ & RS $2.083,33$ & RS 1.166,67 & RS $3.250,00$ & R\$ $125.792,83$ & RS $1.972,63$ & RS $1.277,65$ & RS $3.250,28$ \\
\hline so & RS $119.384,39$ & RS 2.036,36 & RS $1.214,21$ & RS $3.250,57$ & RS $95.833,33$ & RS 2.083,33 & RS 979,17 & RS $3.062,50$ & RS $107.608,86$ & RS $2.059,85$ & RS $1.096,69$ & RS $3.156,53$ \\
\hline 51 & RS $117.327,66$ & RS $2.056,72$ & RS $1.193,84$ & RS $3.250,57$ & RS $93.750,00$ & RS 2.083,33 & RS 958,33 & RS $3.041,67$ & RS $105.538,83$ & RS 2.070,03 & RS $1.076,09$ & RS $3.146,12$ \\
\hline 52 & RS $115 \cdot 250,37$ & RS $2.077,29$ & RS $1.173,28$ & RS $3.250,57$ & RS $91.666,67$ & RS 2.083,33 & RS 937,50 & RS $3.020,83$ & RS $103.458,52$ & RS $2.080,31$ & RS $1.055,39$ & RS $3.135,70$ \\
\hline 53 & RS $113.152,31$ & RS $2.098,06$ & RS 1.152,50 & RS $3.250,57$ & RS $89.583,33$ & RS 2.083,33 & RS 916,67 & RS $3.000,00$ & RS $101.367,82$ & RS $2.090,70$ & RS $1.034,59$ & R\$ $3.125,28$ \\
\hline 60 & RS $97.866,50$ & RS 2.249,41 & RS $1.001,16$ & RS $3.250,57$ & RS $75.000,00$ & RS 2.083,33 & RS 770,83 & RS $2.854,17$ & RS 86.433,25 & RS $2.166,37$ & RS 886,00 & RS $3.052,37$ \\
\hline 70 & RS $74.097,37$ & RS $2.484,75$ & RS 765,82 & RS $3.250,57$ & RS $54.166,67$ & RS 2.083,33 & RS 562,50 & RS $2.645,83$ & RS $64.132,02$ & RS $2.284,04$ & RS 664,16 & RS $2.948,20$ \\
\hline 80 & RS $47.841,45$ & RS $2.744,71$ & RS 505,86 & RS $3.250,57$ & RS $33.333,33$ & RS 2.083,33 & RS 354,17 & RS $2.437,50$ & RS $40.587,39$ & RS $2.414,02$ & RS 430,01 & RS $2.844,03$ \\
\hline 90 & RS $18.838,59$ & RS $3.031,86$ & RS 218,70 & RS $3.250,57$ & RS $12.500,00$ & RS $2.083,33$ & RS 145,83 & RS 2.229,17 & RS $15.669,30$ & RS $2.557,60$ & RS 182,27 & RS $2.739,87$ \\
\hline 95 & RS $3.218,38$ & RS 3.186,52 & RS 64,05 & RS 3.250,57 & RS 2.083,33 & RS 2.083,33 & RS 41,67 & RS $2.125,00$ & R\$ $2.650,86$ & R\$ 2.634,93 & RS 52,86 & RS $2.687,78$ \\
\hline 96 & RS & RS $3.218,38$ & RS 32,18 & RS $3.250,57$ & RS 0,00 & RS $2.083,33$ & RS 20,83 & RS $2.104,17$ & RS 0,00 & RS $2.650,86$ & RS 26,51 & RS $2.677,37$ \\
\hline & TOTAL & RS $200.000,00$ & RS $112.054,56$ & RS $312.054,56$ & . & RS $200.000,00$ & RS $97.000,00$ & RS $297.000,00$ & . & RS $200.000,00$ & RS $104.527,28$ & RS $304.527,28$ \\
\hline
\end{tabular}

Primeiro, vamos analisar o ponto onde as prestações se igualam.

$$
\begin{aligned}
& \left.R^{(P)}=3250,57 \text { constante }\right) \\
& R^{(C)}=A^{(C)}[1+i(n-t+1)]
\end{aligned}
$$

Fazendo $\mathrm{R}^{(\mathrm{P})}=\mathrm{R}_{\mathrm{t}}^{(\mathrm{C})}$, temos:

$$
R^{(P)}=A^{(C)}[1+i(n-t+1)]
$$

$$
\begin{gathered}
3250,57=2083,33[1+0,01(96-t+1)]=4104,16-3250,57-20,83 t \\
t=\frac{853,59}{20,83} \cong 40,97
\end{gathered}
$$


Esses dados podem ser comprovados na Tabela $B$, Para $t=40$, a prestação correspondente ao SAC é superior ao valor correspondente ao Sistema Price, já para $\mathrm{t}=41$, a prestação do SAC é ligeiramente menor que a do Sistema Price.

Agora analisaremos o ponto em que as amortizações são iguais.

$$
\begin{aligned}
A_{t}^{(P)} & =A_{1}^{(P)}(1+i)^{t-1} \\
A^{(C)} & =2083,33(\text { constante }) \\
A_{\mathbf{1}}^{(P)}=R^{(P)}-i \times P_{\mathbf{0}} & =3250,57-0,01 \times 200000,00=1250,57
\end{aligned}
$$

Fazendo $A^{(\mathrm{C})}=A_{t}^{(P)}$, temos:

$$
\begin{aligned}
& 2083,33=1250,57(1,01)^{t-1} \\
& (1,01)^{t-1}=1,66590 \\
& t-1=51,25 \rightarrow t=52,25
\end{aligned}
$$

Podemos observar na Tabela $\mathrm{B}$, que, para $\mathrm{t}=52$, a amortização correspondente ao Sistema Price é inferior à do SAC, já para $\mathrm{t}=53$, a amortização do Sistema Price é superior à do SAC.

Com relação ao SAM, observamos, na Tabela $\mathrm{B}$, que os seus valores estão entre os do Sistema Price e SAC. As prestações do SAM decrescem linearmente, enquanto as suas amortizações crescem de forma exponencial - visto que os seus valores são a média aritmética entre uma constante (SAC) e outra que cresce exponencialmente (Sistema Price).

Nos sistemas trabalhados acima, pudemos perceber, na Tabela B, que no SAC os saldos devedores decrescem mais rapidamente, atingindo 50\% do saldo (nesse caso, R\$ 100.000,00), após o pagamento de $50 \%$ das prestações (igual a 48 prestações), o que significa que o mutuário terá juros mais baixos tendo, consequentemente, prestações mais baixas também. Já na Tabela Price, o saldo devedor terá a sua metade liquidada somente na $60^{\mathrm{a}}$ prestação, que corresponde a $62,5 \%$ do total, o que leva o mutuário a pagar juros mais altos por mais tempo, além de as amortizações na Tabela Price crescerem exponencialmente, fazendo com que o mutuário pague um valor maior, no final do empréstimo. O SAM só terá a metade do seu saldo devedor pago na $54^{\mathrm{a}}$ prestação, o correspondente a $57 \%$ do total.

Por fim, observando a Tabela B, verifica-se que o total de juros cobrado nos três sistemas é diferente, sendo que o SAC tem os juros mais baixos, a Tabela Price tem os juros mais altos e, em uma posição intermediária, vem o SAM, com juros um pouco mais elevados que os do SAC, porém mais baixos que os do Sistema Price.

\section{Considerações Finais}


Durante a pesquisa, foi possível observar como funciona cada um dos três sistemas que nos dispusemos a estudar: o Sistema Price, o SAC e o SAM. Desses três, foi possível extrair vantagens e desvantagens.

Uma das principais desvantagens do SAC e do SAM é que suas prestações iniciais são mais altas que as do Sistema Price (que tem suas prestações constantes), mas, em contrapartida, verificamos que uma de suas vantagens é que, após a metade das prestações, há uma queda significativa no valor das prestações, o que deixaria o mutuário com a renda menos comprometida.

Outra vantagem do SAC são as amortizações; por constantes, isso é, possuírem um valor fixo, o capital abaixa mais rápido, permitindo que se paguem menos juros, a cada prestação.

Apesar do Sistema Price ter o valor de suas prestações constante, as parcelas de suas amortizações iniciais são pequenas, o que gera o pagamento de um valor muito alto de juros, por isso, nesse sistema, pagam-se mais juros no final do plano de financiamento.

Assim, apesar de toda a complexidade das fórmulas e dos cálculos, os sistemas de amortização apresentados neste estudo são de fácil compreensão para as pessoas que desejarem/necessitarem fazer um financiamento ou pedir empréstimo em instituições financeiras. Nosso estudo sugeriu que, com planejamento e organização, é possível ter controle dos juros de uma dívida e manter a vida financeira em ordem.

\section{Referências}

ARAÚJO, Carlos Roberto Vieira. Matemática Financeira: uso das minicalculadoras HP- 12C e HP-19BII. São Paulo: Atlas,1993.

GIMENES, Cristiano Marchi. Matemática Financeira com HP 12C e Excel. São Paulo: Pearson Prentice Hall, 2010.

JARES, X. R. Educar para a Verdade e para a Esperança: em tempos de globalização, guerra preventiva e terrorismo. Porto Alegre: Artmed, 2005.

MATHIAS, Washington Franco et al. Matemática Financeira. 3.ed. São Paulo: Atlas, 2002.

SAMANEZ, Carlos Patricio. Matemática Financeira: aplicações à análise de investimentos. São Paulo: Prentice Hall, 2002.

SOBRINHO, José Dutra Vieira. Matemática Financeira. 7.ed. São Paulo: Atlas, 2000.

Submetido em janeiro de 2015

Aprovado em abril de 2015 